\title{
Sharing an increase of the rent fairly
}

\author{
Rodrigo A. Velez* \\ ${ }^{b}$ Department of Economics, Texas A\&M University, College Station, TX 77843 USA
}

December 1st, 2015

\begin{abstract}
We characterize the family of non-contestable budget-monotone rules for the allocation of objects and money as those obtained by maximizing a min social welfare function among all non-contestable allocations. We provide three additional seemingly independent approaches to construct these rules. We present three applications of this characterization. First, we show that one can "rectify" any non-contestable rule without losing non-contestability. Second, we characterize the preferences that admit, for each budget, a non-contestable allocation satisfying a minimal or maximal individual consumption of money constraint. Third, we study continuity properties of the non-contestable correspondence.
\end{abstract}

JEL classification: D61; D63; D70.

Keywords: solidarity; allocation of objects and money; non-contestable allocations.

\section{Introduction}

A group of roommates pay the rent of their apartment in such a way that no roommate prefers the room of another roommate if she has to pay the other roommate's share of the rent. The landlord increases the rent. This paper characterizes the ways in which the roommates can redistribute the house and rent payments, so they all, in welfare terms, contribute to the higher rent without compromising the initial equitability property of the allocation.

Formally, we study an assignment problem with money. There are $n$ agents who are endowed with $n$ objects. Agents consume exactly one object and an

${ }^{*}$ Thanks to William Thomson for useful comments and
discussions.
https://sites.google.com/site/rodrigoavelezswebpage/home


amount of an infinitely divisible good that we refer to as money. We assume that, ceteris paribus, more money is better for each agent. We refer to the aggregate consumption of money at an allocation as its budget. This model accommodates the allocation of rooms and rent described above, the division of a partnership, and the allocation of tasks and salary among workers.

We study systematic ways of making recommendations for each possible budget. We refer to each such a function as a "rule." We axiomatically study rules. We require two axioms. The first is non-contestabilty, i.e., no agent should prefer the allotment of another agent to her own (Foley, 1967). ${ }^{1}$ This axiom has been central in the theory of equitable allocation (see Thomson, 2010, for a survey). It captures the idea of equal opportunity (Kolm, 1971) and precludes a form of clear-cut bias (Varian, 1974). The second axiom is budget-monotonicity, i.e., the welfare of each agent should be an increasing function of the budget (Moulin and Thomson, 1988). This axioms captures the idea of solidarity when budget changes. The roommates case illustrates it. If the rent to be collected increases, agents should all be responsible for the change and thus be affected in a comparable way. Since money is desirable to each agent, this leads to the requirement that each agent's welfare decreases as the rent to be collected increases.

Alkan et al. (1991) introduce four approaches to construct non-contestable budget-monotone rules. The first is to fix a continuous representation of preferences and for each budget maximize the min social welfare functional (swf) among all non-contestable allocations. The second is again to fix a continuous representation of preferences and for each budget minimize the max' swf among all non-contestable allocations. The third is to fix a list of continuous and increasing functions, one for each object, and then for each budget maximize the minimal transformed consumption of money among all noncontestable allocations. The fourth is again to fix a list of continuous and increasing functions, one for each object, and then for each budget minimize the maximal transformed consumption of money among all non-contestable allocations. Our main result, Theorem 1, states that each of these four approaches spans the whole family of non-contestable budget-monotone rules. Thus, these seemingly independent constructions are actually equivalent: by choosing the parameters of one of these rules, one is implicitly choosing the parameters for the other constructions.

Our contribution goes beyond showing that each of the four constructions above are equivalent. Alkan et al. (1991)'s proof that these rules are budget-

\footnotetext{
${ }^{1}$ This axioms is commonly referred to as "no-envy." See Velez (2015) for a discussion why "non-contestability" reflects better the normative content of the axiom.
} 
monotone is based on linear programming and linear approximation techniques. We present a new constructive proof of this result, which is of independent interest. Indeed, we show that one can obtain budget-monotonicity properties of the non-contestable set directly from the existence of non-contestable allocations results available in the literature (e.g. Svensson, 1983, 1987; Maskin, 1987; Velez, 2015) without using approximation techniques.

We present three applications of Theorem 1. First, we show how one can "rectify" a non-contestable rule that fails to be budget-monotone. A rule is budget monotone at a certain budget, if each agent is worse off at each lower budget and better off at each higher budget. We show that one can "iron" any non-contestable rule preserving both non-contestability and the rule's recommendations for budgets at which it is budget-monotone. That is, given an arbitrary non-contestable rule, say $f$, one can find a non-conestable rule that is "weakly budget-monotone" and coincides with $f$ for the budgets at which $f$ is budget-monotone (Theorem 3 ). Then, we show that given an arbitrary noncontestable rule, say $f$, and a positive $\delta$, one can find a non-contestable rule that is budget-monotone and coincides with $f$ on the budgets at which $f$ is budget-monotone up to a set that can be covered by a set of Lebesgue measure $\delta$ (Theorem 4). Our second application is to characterize the set of preferences that admit for each budget a non-contestable allocation satisfying a minimum or maximum individual consumption of money constraint (Proposition 1). Our third application is to show that the non-contestable correspondence not only is continuous in welfare space, but also admits a continuous selection, a feature that is usually difficult to guarantee for a correspondence that is not convex (Michael, 1956). This allows us to show the existence of noncontestable budget-monotone efficient rules in the extension of our model where there are more objects than agents (Sec. 4.3).

Our approach to study rules and their properties, as opposed to studying isolated allocation problems, can be traced back to Thomson (1983). Previous literature has explored the performance of allocation rules when one considers other dimensions of assignment problems with money as variable. Most notably, Alkan et al. (1991) and Alkan (1994) study solidarity notions under the arrival of objects; Alkan (1994) and Tadenuma and Thomson (1995) study solidarity notions under the arrival of new agents; and Tadenuma and Thomson (1991) study the consistency axiom, which can be interpreted as a form of solidarity when allocations have to be reassessed for a subset of agents with their corresponding initial allotments (Thomson, 2012). 


\section{Model}

\subsection{Environment}

Let $N$ be a set of $n$ agents and $A$ a set of $n$ objects. Generic agents are $i, j$, and generic objects are $\alpha, \beta$. Each agent consumes one object and an amount of a perfectly divisible good we refer to as money. Agent $i$ 's generic consumption bundle is $\left(x_{i}, \alpha\right)$ where $x_{i} \in \mathbb{R}$ and $\alpha \in A$. Each agent has complete and transitive preferences on consumption bundles. Agent $i$ 's preference is $\boldsymbol{R}_{\boldsymbol{i}}$ and fixed throughtout. The symmetric and asymmetric parts of $R_{i}$ are denoted by $\boldsymbol{I}_{\boldsymbol{i}}$ and $\boldsymbol{P}_{\boldsymbol{i}}$ respectively. Preferences are money monotone: ceteris paribus, each agent prefers a higher consumption of money. Preferences also satisfy the compensation assumption: for each bundle $\left(x_{i}, \alpha\right)$ and each object $\beta$, there is $x_{i}^{\prime}$ such that $\left(x_{i}, \alpha\right) I_{i}\left(x_{i}^{\prime}, \beta\right){ }^{2}$ The profile of preferences is $\boldsymbol{R} \equiv\left(\boldsymbol{R}_{\boldsymbol{i}}\right)_{\boldsymbol{i} \in \boldsymbol{N}}$.

An allocation is a pair $\boldsymbol{z} \equiv(\boldsymbol{x}, \boldsymbol{\mu})$ where $x \equiv\left(x_{i}\right)_{i \in N} \in \mathbb{R}^{n}$ and $\mu: N \rightarrow A$ is a bijection. Agent $i$ 's bundle at $z$ is $z_{i} \equiv\left(x_{i}, \mu_{i}\right)$, where $x_{i}$ is her consumption of money and $\mu_{i}$ her assigned object. The set of allocations is $Z$. We denote the set of allocations whose aggregate consumption of money is $m \in \mathbb{R}$, i.e., $(x, \mu)$ such that $\sum_{i \in N} x_{i}=m$, by $\boldsymbol{Z}_{\boldsymbol{m}}$. Each agent's preferences induce a preference on allocations: for each pair $\left\{z, z^{\prime}\right\} \subseteq Z, z R_{i} z^{\prime}$ if $z_{i} R_{i} z_{i}^{\prime}$. For an allocation $z \equiv$ $(x, \mu)$ we denote the consumption of money of the agent who receives object $\alpha \in A$ at $z$ by $\boldsymbol{x}_{\boldsymbol{\alpha}}$.

For each $i \in N$, each pair $\{\alpha, \beta\} \in A$, and each $x_{\alpha} \in \mathbb{R}$, let $\boldsymbol{q}_{i}\left(\boldsymbol{x}_{\boldsymbol{\alpha}}, \boldsymbol{\alpha}, \boldsymbol{\beta}\right) \in \mathbb{R}$ be the amount such that $\left(x_{\alpha}, \alpha\right) I_{i}\left(q_{i}\left(x_{\alpha}, \alpha, \beta\right), \beta\right)$. For each $m \in \mathbb{R}$ and each $z \equiv(x, \mu) \in Z_{m}$, let $\boldsymbol{d}_{\boldsymbol{i} \boldsymbol{j}}(z) \equiv q_{i}\left(x_{i}, \mu_{i}, \mu_{j}\right)-x_{j}$. For each $x, y \in \mathbb{R}^{2}, \operatorname{seg}(\boldsymbol{x}, \boldsymbol{y}) \equiv$ $\left\{z \in \mathbb{R}^{2}: z=\lambda x+(1-\lambda) y, \lambda \in[0,1]\right\}$ is the segment from $x$ to $y$.

\subsection{Socially desirable allocation rules}

We search for socially desirable systematic ways to provide recommendations for each possible configuration of resources in our environment. We refer to such an object as a rule, i.e., a function that assigns to each possible aggregate consumption of money, say $m$, an allocation in $Z_{m}$. We denote the generic rule by $f: \mathbb{R} \rightarrow Z$. We denote the consumption bundle assigned to agent $i \in N$ at $f(m)$ by $f_{i}(m)$.

By studying rules we can evaluate our recommendations with two different perspectives. First, we can formulate axioms, i.e., properties of allocations that can be justified in normative terms. The following two axioms capture the

\footnotetext{
${ }^{2}$ Money-monotonicity and the compensation assumption imply continuity.
} 
idea that, in welfare terms, an allocation should not be unambiguously dominated by another feasible allocation and should not be unambiguously biased towards any agent:

$z \in Z_{m}$ is efficient if there is no $z^{\prime} \in Z_{m}$ such that for each $i \in N, z^{\prime} R_{i}, z$ and for at least one $j \in N, z^{\prime} P_{j} z ; z \in Z$ is non-contestable if for each pair $\{i, j\} \subseteq N, z_{i} R_{i} z_{j}$ (Foley, 1967; Varian, 1974).

In our environment, non-contestable allocations exist (Svensson, 1983; Alkan et al., 1991; Velez, 2015) and are efficient (Svensson, 1983). We denote the set of non-contestable allocations by $\boldsymbol{F}$ and the set of non-contestable allocations with aggregate consumption of money $m$ by $\boldsymbol{F}_{\boldsymbol{m}}$. We say that a rule $\boldsymbol{f}$ is noncontestable if for each $m, f(m)$ is non-contestable.

Second, by studying rules instead of individual allocations we can articulate the idea of "solidarity," i.e., given a change in the conditions that describe the resource allocation problem, agents should share the welfare gains or losses in comparable ways. Since interpersonal comparisons of utility are difficult, an uncontroversial requirement is that each agent's welfare change in the same direction (Thomson, 1983). In our model, in which the aggregate consumption of money is the only parameter that can change and money is desirable to all agents, this solidarity principle takes a simple form:

A rule $f$ is budget-monotone at $\boldsymbol{m} \in \mathbb{R}$ if (1) for each $l<m$, each agent prefers $f(m)$ to $f(l)$; and (2) for each $h>m$, each agent prefers $f(h)$ to $f(m)$; $f$ is budget-monotone if it is budget-monotone at each $m \in \mathbb{R}$.

We now introduce five families of rules based on intuitive criteria of distributive justice. The first four families allow us to make a simple description of the non-contestable and budget-monotone rules. The other two families of rules are non-contestable but violate budget-monotonicity; in Sec. 4.1 we show how we can reconcile these intuitive approaches to fair allocation with budgetmonotonicity.

An non-contestable rule $f$ is:

- Value-Maxmin (Alkan et al., 1991): ${ }^{3}$ if there is a continuous representation of preferences $u \equiv\left(u_{i}\right)_{i \in N}$ such that for each $m$,

$$
f(m) \in \underset{z \in F_{m}}{\operatorname{argmax}}\left\{\min _{i \in N} u_{i}\left(z_{i}\right)\right\} .
$$

\footnotetext{
${ }^{3}$ This family of rules can be seen as the implementation of the Rawlsian principle of distributive justice (Rawls, 1972) constrained by efficiency and no-envy.
} 
- Money-Maxmin (Alkan et al., 1991; Velez, 2011): if there is a profile of continuous strictly increasing functions $g \equiv\left(g_{\alpha}\right)_{\alpha \in A}$ where for each $\alpha$, $g_{\alpha}: \mathbb{R} \rightarrow \mathbb{R}$ and for each $m$,

$$
f(m) \in \underset{z \equiv(x, \mu) \in F_{m}}{\operatorname{argmax}}\left\{\min _{\alpha \in A} g_{\alpha}\left(x_{\alpha}\right)\right\} .
$$

- Value-Minmax (Alkan et al., 1991): if there is a continuous representation of preferences $u \equiv\left(u_{i}\right)_{i \in N}$ such that for each $m$,

$$
f(m) \in \underset{z \in F_{m}}{\operatorname{argmin}}\left\{\max _{i \in N} u_{i}\left(z_{i}\right)\right\} .
$$

- Money-Minmax (Alkan et al., 1991; Velez, 2011): if there is a profile of continuous strictly increasing functions $g \equiv\left(g_{\alpha}\right)_{\alpha \in A}$ where for each $\alpha$, $g_{\alpha}: \mathbb{R} \rightarrow \mathbb{R}$ and for each $m$,

$$
f(m) \in \underset{z \equiv(x, \mu) \in F_{m}}{\operatorname{argmin}}\left\{\max _{\alpha \in A} g_{\alpha}\left(x_{\alpha}\right)\right\} .
$$

- Utilitarian: if there is a continuous representation of preferences $u \equiv$ $\left(u_{i}\right)_{i \in N}$ such that for each $m$,

$$
f(m) \in \underset{z \in F_{m}}{\operatorname{argmax}}\left\{\sum_{i \in N} u_{i}\left(z_{i}\right)\right\} .
$$

- Equal-compensation rule (Tadenuma and Thomson, 1995): if for each $m$,

$$
f(m) \in\left\{z \in F_{m}: \text { for each } z^{\prime} \in F_{m}, \min _{i, j \in N, i \neq j} d_{i j}(z) \geq \min _{i, j \in N, i \neq j} d_{i j}\left(z^{\prime}\right)\right\} .
$$

\section{Budget-monotone rules}

Each of the families of value-maxmin, money-maxmin, value-minmax, and money-minmax rules defined in Sec. 2.2 coincides with the family of non-contestable budget-monotone rules.

Theorem 1. Let $f$ be non-contestable. The following are equilivalent:

1. $f$ is budget-monotone

2. $f$ is Value-Maxmin. 
3. $f$ is Money-Maxmin.

4. $f$ is Value-Minmax.

5. $f$ is Money-Minmax.

We first prove that statement 1 implies statements 2-5 in Theorem 1.

Proof. Let $f$ be non-contestable and budget monotone. We first prove that $f$ is value-maxmin and value-minmax. We construct a profile of continuous utility functions $u \equiv\left(u_{i}\right)_{i \in N}$ representing $R$ such that for each $m$, and each pair $\{i, j\} \subseteq N, u_{i}\left(f_{i}(m)\right)=u_{j}\left(f_{j}(m)\right)$. We claim that $f(m)$ is a value-maxmin rule for $u$. Suppose by contradiction that there is $m \in \mathbb{R}$ and $z \in F_{m}$ such that $\min _{i \in N} u_{i}\left(z_{i}\right)>\min _{i \in N} u_{i}\left(f_{i}(m)\right)$. Then, for each $i \in N, u_{i}\left(z_{i}\right)>u_{i}\left(f_{i}(m)\right)$. Thus, $f(m)$ is not efficient. Thus, $f(m) \notin F$ (Svensson, 1983). This is a contradiction. A symmetric argument shows that $f$ is a value-minmax rule for $u$. For each $i \in N$, let $w_{i}$ be a continuous representation of $R_{i}$ and $v_{i} \equiv w_{i} \circ f_{i}$. We claim that each $v_{i}$ is a continuous function. Let $m \in \mathbb{R}$ and $\left\{m_{k}\right\}_{k \in \mathbb{N}}$ be a sequence converging to $m$ as $k \rightarrow \infty$. We prove that as $k \rightarrow \infty, v_{i}\left(m_{k}\right) \rightarrow v_{i}(m)$. Since $\left\{m_{k}\right\}_{k \in \mathbb{N}}$ converges, it is bounded. Thus, since $f$ is budget-monotone, $\left\{v_{i}\left(m_{k}\right)\right\}_{k \in \mathbb{N}}$ is bounded. Thus, it suffices to show that if $\left\{v_{i}\left(m_{k}\right)\right\}_{k \in \mathbb{N}}$ converges, its limit is $v_{i}(m)$. Now, since $\left\{m_{k}\right\}_{k \in \mathbb{N}}$ is bounded and $f$ is non-contestable, $\left\{f\left(m_{k}\right)\right\}_{k \in \mathbb{N}}$ is bounded in $\mathbb{R}^{N} \times A^{N}$ (endowed with the box topology). Select a subsequence of $\left\{f\left(m_{k}\right)\right\}_{k \in \mathbb{N}}$ that converges as $k \rightarrow \infty$ and let $z \equiv(x, \mu)$ be its limit. Since each $v_{i}$ is continuous, as $k \rightarrow \infty, v_{i}\left(m_{k}\right) \rightarrow w_{i}\left(z_{i}\right)$. Since preferences are continuous, then $z \in F$. Since each subsequence of $\left\{m_{k}\right\}_{k \in N}$ converges to $m$, then $\sum_{i \in N} x_{i}=m$ and $z \in F_{m}$. There are two cases.

Case 1: there is a subsequence of $\left\{m_{k}\right\}_{k \in \mathbb{N}},\left\{\widetilde{m}_{k}\right\}_{k \in \mathbb{N}}$, such that for each $k \in$ $\mathbb{N}, \widetilde{m}_{k} \leq m$. Then, for each $k \in \mathbb{N}, v_{i}\left(\widetilde{m}_{k}\right) \leq v_{i}(m)$. Thus, for each $i \in N, w_{i}\left(z_{i}\right) \leq$ $v_{i}(m)$. Thus, for each $i \in N, w_{i}\left(z_{i}\right)=v_{i}(m)=w_{i}\left(f_{i}(m)\right)$, for otherwise $z$ is neither efficient nor non-contestable.

Case 2: there is a subsequence of $\left\{m_{k}\right\}_{k \in \mathbb{N}},\left\{\widetilde{m}_{k}\right\}_{k \in \mathbb{N}}$, such that for each $k \in$ $\mathbb{N}, \widetilde{m}_{k} \geq m$. Then, for each $k \in \mathbb{N}, v_{i}\left(\widetilde{m}_{k}\right) \geq v_{i}(m)$. Thus, for each $i \in N, w_{i}\left(z_{i}\right) \geq$ $v_{i}(m)$. Thus, for each $i \in N, w_{i}\left(z_{i}\right)=v_{i}(m)=w_{i}\left(f_{i}(m)\right)$, for otherwise $f(m)$ is neither efficient nor non-contestable.

Let $i \in N$ and $\left(x_{i}, \alpha\right) \in \mathbb{R} \times A$. Let $u_{i}\left(x_{i}, \alpha\right) \equiv m$ such that $w_{i}\left(x_{i}, \alpha\right)=w_{i}(f(m))$. We claim that $u_{i}$ is well defined. Since preferences are money-monotone and satisfy the compensation assumption, there is $l \in \mathbb{R}$ such that for each $z \in$ $F_{l}, w_{i}\left(z_{i}\right) \leq\left(x_{i}, \alpha\right)$; symmetrically, there is $h \in \mathbb{R}$ such that for each $z \in F_{h}$, $w_{i}\left(x_{i}, \alpha\right) \leq w_{i}\left(z_{i}\right) \leq$. Thus, $v_{i}(l) \leq w_{i}\left(z_{i}\right) \leq v_{i}(h)$. Since $v_{i}$ is continuous, there 
is $m \in \mathbb{R}$ such that $v_{i}(m)=w_{i}(m)$. Since $f$ is budget-monotone, $v_{i}$ is strictly increasing and such $m$ is unique. We claim that $u_{i}$ represents $R_{i}$. Let $\left(x_{i}, \alpha\right)$ and $\left(x_{i}^{\prime}, \alpha^{\prime}\right)$ be such that $\left(x_{i}^{\prime}, \alpha^{\prime}\right) R_{i}\left(x_{i}, \alpha\right)$. We claim that $u_{i}\left(x_{i}^{\prime}, \alpha^{\prime}\right) \geq u_{i}\left(x_{i}, \alpha\right)$. Suppose by means of contradiction that $u_{i}\left(x_{i}, \alpha\right)>u_{i}\left(x_{i}^{\prime}, \alpha^{\prime}\right)$. Since $f$ is budgetmonotone, $w_{i}\left(f_{i}\left(u_{i}\left(x_{i}, \alpha\right)\right)\right)>w_{i}\left(f\left(u_{i}\left(x_{i}^{\prime}, \alpha^{\prime}\right)\right)\right)$. Thus, $w_{i}\left(x_{i}, \alpha\right)>w_{i}\left(x_{i}^{\prime}, \alpha^{\prime}\right)$. This contradicts $\left(x_{i}^{\prime}, \alpha^{\prime}\right) R_{i}\left(x_{i}, \alpha\right)$. Now, let $\left(x_{i}, \alpha\right)$ and $\left(x_{i}^{\prime}, \alpha^{\prime}\right)$ be such that $u_{i}\left(x_{i}^{\prime}, \alpha^{\prime}\right) \geq$ $u_{i}\left(x_{i}, \alpha\right)$. Since $f$ is budget-monotone, $w_{i}\left(f_{i}\left(u_{i}\left(x_{i}^{\prime}, \alpha^{\prime}\right)\right)\right) \geq w_{i}\left(f\left(u_{i}\left(x_{i}, \alpha\right)\right)\right)$. Thus, $\left(x_{i}^{\prime}, \alpha^{\prime}\right) R_{i}\left(x_{i}, \alpha\right)$. By construction, for each $m \in \mathbb{R}$ and each pair $\{i, j\} \subseteq N$, $u_{i}\left(f_{i}(m)\right)=u_{j}\left(f_{j}(m)\right)=m$. Finally, we claim that $u_{i}$ is continuous. Let $\left\{\left(x_{i}^{k}, \alpha^{k}\right)\right\}_{k \in \mathbb{N}}$ be a convergent sequence in $\mathbb{R} \times A$ and let $\left(x_{i}, \alpha\right)$ be its limit as $k \rightarrow \infty$. Without loss of generality we can assume that the sequence $\left\{\alpha^{k}\right\}_{k \in \mathbb{N}}$ is constant. We claim that $u_{i}\left(x_{i}^{k}, \alpha\right)$ converges to $u_{i}\left(x_{i}, \alpha\right)$. For each $k \in \mathbb{N}$, let $m_{k} \equiv u_{i}\left(x_{i}^{k}, \alpha\right)$. Since $\left\{x_{i}^{k}\right\}_{k \in \mathbb{N}}$ is convergent, it is bounded. Since preferences are money-monotone, $\left\{m_{k}\right\}_{k \in \mathbb{N}}$ is bounded. Thus, we can suppose without loss of generality that $\left\{m_{k}\right\}_{k \in \mathbb{N}}$ is convergent and prove that its limit is $u_{i}\left(x_{i}, \alpha\right)$. Let $m$ be the limit as $k \rightarrow \infty$ of $\left\{m_{k}\right\}_{k \in \mathbb{N}}$. For each $k \in \mathbb{N}$, let $z^{k} \equiv f\left(m_{k}\right)$. Since $\left\{m_{k}\right\}_{k \in \mathbb{N}}$ is bounded, so is $\left\{z^{k}\right\}_{k \in \mathbb{N}}$. Thus, one can select a convergent subsequence of $\left\{z^{k}\right\}_{k \in \mathbb{N}}$. Let $z$ be the limit of such a convergent subsequence. Since $w_{i}$ is continuous, $\left\{w_{i}\left(z_{i}^{k}\right)\right\}_{k \in \mathbb{N}}$ converges as $k \rightarrow \infty$ to $w_{i}\left(z_{i}\right)$. Since for each $k \in$ $\mathbb{N}, w_{i}\left(z_{i}^{k}\right)=w_{i}\left(x_{i}^{k}, \alpha\right)$, then $\left\{w_{i}\left(x_{i}^{k}, \alpha\right)\right\}_{k \in \mathbb{N}}$ converges as $k \rightarrow \infty$ to $w_{i}\left(x_{i}, \alpha\right)$. Thus, $w_{i}\left(z_{i}\right)=w_{i}(x, \alpha)$. From our argument above, $w_{i}\left(z_{i}\right)=w_{i}\left(f_{i}(m)\right)$. Thus, $w_{i}\left(x_{i}, \alpha\right)=w_{i}\left(f_{i}(m)\right)$ and $u_{i}\left(x_{i}, \alpha\right)=m$.

We now prove that $f$ is money-maxmin and money-minmax. We construct a profile of continuous increasing functions $g \equiv\left(g_{\alpha}\right)_{\alpha \in A}$ where for each $\alpha, g_{\alpha}$ : $\mathbb{R} \rightarrow \mathbb{R}$, with the following property. For each $m$ and each pair $\{\alpha, \beta\} \subseteq A$, if $f(m) \equiv(x, \mu)$, then $g_{\alpha}\left(x_{\alpha}\right)=g_{\beta}\left(x_{\beta}\right)$. We claim that $f$ is a money-maxmin rule for such a profile of functions. Suppose by contradiction that there is $m \in \mathbb{R}$ and $z \equiv(y, \mu) \in F_{m}$ such that $\min _{\alpha \in A} g_{\alpha}\left(y_{\alpha}\right)>\min _{\alpha \in A} g_{\alpha}\left(x_{\alpha}\right)$. Since each $g_{\alpha}$ is increasing, then for each $\alpha \in A, y_{\alpha}>x_{\alpha}$. Thus, $\sum_{\alpha \in A} y_{\alpha}>m$. This is a contradiction. A symmetric argument shows that $f$ is a money-minmax rule for $g$. For each $\alpha \in A$ and each $m \in \mathbb{R}$, let $y_{\alpha}(m) \equiv x_{\alpha}$ where $f(m)=(x, \mu)$. Since $f$ is budget-monotone, by the Decomposition Lemma (Alkan et al., 1991), which we state in the Appendix, each $y_{\alpha}$ is a strictly increasing function. We claim that each $y_{\alpha}$ is continuous. Let $\left\{m_{k}\right\}_{k \in \mathbb{N}}$ be a convergent sequence and $m$ its limit as $k \rightarrow \infty$. Let $z \equiv(x, \mu) \equiv f(m)$. We prove that $\left\{y_{\alpha}\left(m_{k}\right)\right\}_{k \in \mathbb{N}}$ converges as $k \rightarrow \infty$ to $y_{\alpha}(m)=x_{\alpha}$. Since $\left\{m_{k}\right\}_{k \in \mathbb{N}}$ converges, it is bounded. Thus, since $y_{\alpha}$ is strictly increasing, $\left\{y_{\alpha}\left(m_{k}\right)\right\}_{k \in \mathbb{N}}$ is bounded. Thus, we can suppose without loss of generality that $\left\{y_{\alpha}\left(m_{k}\right)\right\}_{k \in \mathbb{N}}$ is convergent and prove that its limit as $k \rightarrow \infty$ is $y_{\alpha}(m)$. Since $\left\{m_{k}\right\}_{k \in \mathbb{N}}$ is bounded and $f$ is non-contestable, $\left\{f\left(m_{k}\right)\right\}_{k \in \mathbb{N}}$ is bounded in $\mathbb{R}^{N} \times A^{N}$. Let $\left\{\widetilde{z}^{k} \equiv\left(\widetilde{x}^{k}, \widetilde{\mu}^{k}\right)\right\}_{k \in \mathbb{N}}$ be a convergent subsequence of 
$\left\{f\left(m_{k}\right)\right\}_{k \in \mathbb{N}}$ and $\widetilde{z} \equiv(\widetilde{x}, \widetilde{\mu})$ be its limit as $k \rightarrow \infty$. Since preferences are continuous, $\widetilde{z} \in F$. Since as $k \rightarrow \infty, \widetilde{m}_{k} \rightarrow m, \widetilde{z} \in F_{m}$. There are two cases. If there is a convergent subsequence of $\left\{f\left(m_{k}\right)\right\}_{k \in \mathbb{N}},\left\{f\left(\widetilde{m}_{k}\right)\right\}_{k \in \mathbb{N}}$, such that for each $k \in \mathbb{N}$, $\widetilde{m}_{k} \leq m$. Let $\widetilde{z} \equiv(\widetilde{x}, \widetilde{\mu})$ be the limit as $k \rightarrow \infty$ of $\left\{f\left(\widetilde{m}_{k}\right)\right\}_{k \in \mathbb{N}}$. Since $f$ is budgetmonotone, by the Decomposition Lemma, for each $k \in \mathbb{N}, \widetilde{x}^{k} \leq x$. Thus, $\widetilde{x} \leq x$. Since $\sum_{\alpha \in A} x_{\alpha}=\sum_{\alpha \in A} \tilde{x}_{\alpha}=m$, then $\tilde{x}=x$. Since $\left\{y_{\alpha}\left(m_{k}\right)\right\}_{k \in \mathbb{N}}$ is convergent and $\left\{\widetilde{x}_{\alpha}^{k}\right\}_{k \in \mathbb{N}}$ is one of its subsequences, as $k \rightarrow \infty, y_{\alpha}\left(m_{k}\right) \rightarrow \widetilde{x}_{\alpha}=x_{\alpha}$. The argument is symmetric if there is a convergent subsequence of $\left\{f\left(m_{k}\right)\right\}_{k \in \mathbb{N}}$, $\left\{f\left(\widetilde{m}_{k}\right)\right\}_{k \in \mathbb{N}}$, such that for each $k \in \mathbb{N}, \widetilde{m}_{k} \geq m$. Let $\alpha \in A$. For each $x_{\alpha} \in \mathbb{R}$, let $g_{\alpha}\left(x_{\alpha}\right) \equiv m$ such that the consumption of money of the agent who receives object $\alpha$ at $f(m)$ is $x_{\alpha}$. As above, by the Intermediate Value Theorem, $g_{\alpha}$ is well defined. By construction, for each $m$ and each pair $\{\alpha, \beta\} \subseteq A$, if $f(m) \equiv(x, \mu)$, $g_{\alpha}\left(x_{\alpha}\right)=g_{\beta}\left(x_{\beta}\right)=m$. Since $f$ is budget-monotone, each $g_{\alpha}$ is strictly increasing. Finally, we prove that each $g_{\alpha}$ is continuous. Let $\left\{x_{\alpha}^{k}\right\}_{k \in \mathbb{N}}$ be a convergent sequence and let $x_{\alpha}$ be its limit as $k \rightarrow \infty$. We prove that as $k \rightarrow \infty$, $g_{\alpha}\left(x_{\alpha}^{k}\right) \rightarrow g_{\alpha}\left(x_{\alpha}\right)$. Since $\left\{x_{\alpha}^{k}\right\}_{k \in \mathbb{N}}$ converges, it is bounded. Since $\left\{x_{\alpha}^{k}\right\}_{k \in \mathbb{N}}$ is bounded and $g_{\alpha}$ is increasing, $\left\{g_{\alpha}\left(x_{\alpha}^{k}\right)\right\}_{k \in \mathbb{N}}$ is bounded. Thus, it is enough to prove that if $\left\{m_{k} \equiv g_{\alpha}\left(x_{\alpha}^{k}\right)\right\}_{k \in \mathbb{N}}$ converges, its limit as $k \rightarrow \infty$ is $g_{\alpha}\left(x_{\alpha}\right)$. Let $m$ be the limit as $k \rightarrow \infty$ of $\left\{m_{k}\right\}_{k \in \mathbb{N}}$. We proved above that $y_{\alpha}$ is continuous. Thus, as $k \rightarrow \infty, x_{\alpha}^{k}=y_{\alpha}\left(m_{k}\right) \rightarrow y_{\alpha}(m)$. Thus, $y_{\alpha}(m)=x_{\alpha}$. Thus, $g_{\alpha}\left(x_{\alpha}\right)=m$.

We now discuss the proof that each of numerals 2-4 imply numeral 1 in Theorem 1. This was first stated and proved by Alkan et al. (1991). A preference is "piece-wise linear" if it is represented by a function whose marginal utility of money is constant over a finite set of intervals. ${ }^{4}$ Alkan et al. (1991) base their work on linear programming applied to linear preference profiles, the local linearization of piece-wise linear preferences, and the approximation of a continuous preference by piece-wise linear preferences. We present an alternative direct proof based only on the existence of non-contestable allocations for a continuous economy. The key to our proof is the following result. ${ }^{5}$

Theorem 2 (Positive monotonicity). Let $m \in \mathbb{R}, z \in F_{m}$, and $\varepsilon>0$. Then, there is an allocation in $F_{m+\varepsilon}$ that each agent prefers to $z$.

Proof. Let $m \in \mathbb{R}$ and $z \equiv(x, \mu) \in F_{m}$. We first prove that there is $\Delta>0$ such that for each $\delta \in(0, \Delta)$ there is $z^{\delta} \in F_{m+\delta}$ that each agent prefers to $z$. Let $m \in \mathbb{R}$

\footnotetext{
${ }^{4}$ The domain of piece-wise linear preferences can be defined without reference to utility representations by interpolating a finite set of ordered indifference sets.

${ }^{5}$ The Positive Monotonicity Theorem was first stated by Alkan (1994) as a corollary to Alkan et al. (1991)'s results. Our contribution here is to provide a direct proof of it that requires no linear approximation.
} 
and $z \equiv(x, \mu) \in F_{m}$. Let $A_{i}=\left\{\alpha \in A: z_{i} I_{i}\left(x_{\alpha}, \alpha\right)\right\}$ (Fig. 1 (a)). If for each $i \in N$, $A_{i}=A$, the lemma follows from Svensson $(1983,1987) .{ }^{6}$ Suppose that there is $i \in N$ such that $A_{i} \neq A$. For each such $i$ and $\alpha \in A \backslash A_{i}, q_{i}\left(x_{i}, \mu_{i}, \alpha\right)-x_{\alpha}>0$. Let $\Delta \equiv \min \left\{q_{i}\left(x_{i}, \mu_{i}, \alpha\right)-x_{\alpha}: i \in N, \alpha \in A \backslash A_{i}\right\}$. Since $z \in F, \Delta \geq 0$. Since there is $i \in N$ for whom $A \neq A_{i}, \Delta>0$. Let $0<\delta<\Delta$. We prove that there is an allocation $z^{\delta} \in F_{m+\delta}$ that each agent prefers to $z$. We do this by constructing an auxiliary profile of preferences represented by a utility profile that we denote by $v \equiv\left(v_{i}\right)_{i \in N}$. We denote the set of non-contestable allocations for $v$ by $F^{v}$ and the subset of $F^{v}$ with aggregate consumption of money $m \in \mathbb{R}$ by $F_{m}^{v}$. Profile $v$ has the following properties: (1) there is $(y, \sigma) \in F_{\delta}^{v}$ such that $y \gg 0$; (2) if $\left(x+{ }_{A} y\right) \in \mathbb{R}^{N}$ is the vector whose $i$-th component is $x_{\sigma_{i}}+y_{i},\left(x+_{A} y, \sigma\right) \in F_{m+\delta}$. By the Decomposition Lemma, the existence of $v$ proves the lemma.

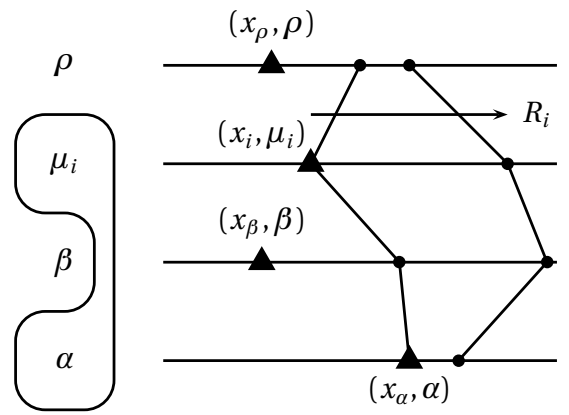

$I_{i}(x, \sigma)$

(a) (b)

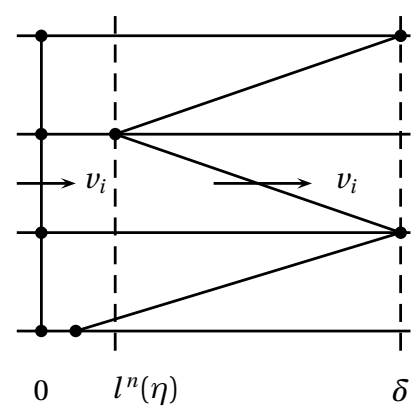

(d)

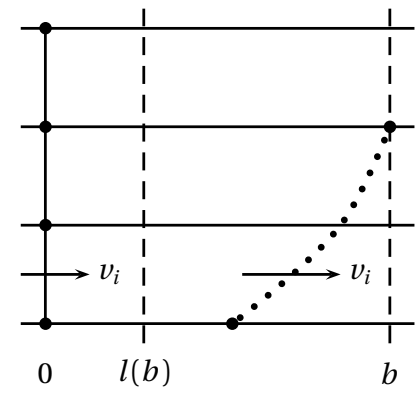

(c) consumption bundle $\left(x_{\alpha}, \alpha\right)$. Indifference sets of preferences in the construction are joined by lines. Arrows represent the direction in which welfare increases.

${ }^{6}$ See also Velez (2015) for a constructive proof. 
Let $u \equiv\left(u_{i}\right)_{i \in N}$ be a continuous numerical representation of $R$. We define $v$. For each $i \in N$, each $b \geq 0$, and each $\alpha \in A_{i}$, let $v_{i}(b, \alpha)=u_{i}\left(x_{\alpha}+b, \alpha\right) .{ }^{7}$ For each $b \geq 0$, let $l(b) \equiv \min \left\{q_{i}\left(x_{\alpha}+b, \alpha, \beta\right)-x_{\beta}: i \in N,\{\alpha, \beta\} \subseteq A_{i}\right\}$. Since for each $i \in N$ and each pair $\{\alpha, \beta\} \subseteq A_{i},\left(x_{\alpha}, \alpha\right) I_{i}\left(x_{\beta}, \beta\right)$, for each $b>0, l(b)>0$. By definition, for each $b \geq 0$, each $a \leq b$, each $i \in N$, and each pair $\{\alpha, \beta\} \subseteq A_{i}$, $v_{i}(b, \alpha) \geq v_{i}(a, \beta)$ (Fig. 1 (c)). Thus, for each $(y, \sigma) \in F^{v}$ such that $y \gg 0$, if there is $i \in N$ and $b \geq 0$ such that $v_{i}\left(l(b), \mu_{i}\right) \geq v_{i}\left(y_{i}, \sigma\right)$, then for each $\beta \in A_{i}, y_{\beta} \leq b$.

Let $\eta \equiv \delta /[n(n+1)]$. For each $i \in N$ and each $\beta \in A \backslash A_{i}$, let $v_{i}(\cdot, \beta)$, be a continuous and strictly increasing function such that: (1) for each $\rho \in A \backslash A_{i}$, $v_{i}(\cdot, \beta)=v_{i}(\cdot, \rho)$; (2) $v_{i}(0, \boldsymbol{\beta})=u_{i}\left(x_{i}, \mu_{i}\right)$; and (3) $v_{i}(\delta, \beta)=\min \left\{u_{i}\left(l^{n}(\eta), \alpha\right): \alpha \in\right.$ $\left.A_{i}\right\}$. (Fig. 1 (d)). For each $i \in N$ and each pair $\{\alpha, \beta\} \subseteq A, v_{i}(0, \alpha)=v_{i}(0, \beta)$. Thus, there is $(y, \sigma) \in F_{\delta}^{v}$ such that $y \geq 0$ (Svensson, 1983, 1987). Let $(y, \sigma) \in F_{\delta}^{v}$. Since $\delta>0,(y, \sigma) \in F_{\delta}^{\nu}$, and for each $i \in N$ and each pair $\{\alpha, \beta\} \subseteq A, v_{i}(0, \alpha)=v_{i}(0, \beta)$, $y \gg 0$. We claim that for each $i \in N, \sigma_{i} \in A_{i}$. Suppose by contradiction (and w.l.o.g.) that $\sigma_{1} \notin A_{1}$. Then, $y_{1} \geq \delta / n$ for there is $i \in N$ such that $y_{i} \geq$ $\delta / n \geq l^{n}(\eta)$. Suppose w.l.o.g. that $\sigma_{2}=\mu_{1}$. Since $(y, \sigma) \in F_{\delta}^{v}, y_{2} \leq l^{n}(\eta)$ and $v_{2}\left(y_{\mu_{2}}, \mu_{2}\right) \leq v_{2}\left(y_{2}, \sigma_{2}\right)$. Now, by construction $v_{2}\left(l^{n}(\eta), \sigma_{2}\right) \leq v_{2}\left(l^{n-1}(\eta), \mu_{2}\right) .^{8}$ Thus, $y_{\mu_{2}} \leq l^{n-1}(\eta)$. If $\mu_{2}=\sigma_{1}$, we have a contradiction to Pareto-efficiency of $(y, \sigma)$ for profile $v$ because if agent 1 receives $\left(l^{n}(\eta), \mu_{1}\right)$ and agent 2 receives $\left(l^{n-1}(\eta), \mu_{2}\right)$, both agents are not worse off and $l^{n}(\eta)+l^{n-1}(\eta) \leq 2 \eta<\delta / n$. Suppose that $\mu_{2} \neq \sigma_{1}$. Renaming the agents if necessary and using successively the same argument as above, we can find $k \in N$ such that for each $j \in\{1, \ldots, k\}$, $v_{j}\left(y_{j}, \sigma_{j}\right) \leq v_{j}\left(l^{n+1-j}(\eta), \mu_{j}\right), \cup_{j=1}^{k} \mu_{j}=\cup_{j=1}^{k} \sigma_{j}$, and $\mu_{k}=\sigma_{1}$. This is a contradiction to Pareto-efficiency of $(y, \sigma)$ for profile $v$ because $\sum_{j=1}^{k} l^{n+1-j}(\eta) \leq n \eta<$ $\delta / n$. Since for each $i \in N, \sigma_{i} \in A_{i}$ and $\delta<\Delta$, for each $i \in N$, each $\alpha \in A_{i}$, and each $\beta \in A \backslash A_{i},\left(x_{\alpha}, \alpha\right) R_{i}\left(x_{\beta}+\delta, \beta\right)$. Thus, at $\left(x+_{A} y, \sigma\right)$ no $i \in N$ prefers the consumption of another agent who receives an object in $A \backslash A_{i}$. Recall that for each $i \in N$, each $u_{i}$ and $v_{i}$ coincide for bundles with objects in $A_{i}$. Thus, at $\left(x+{ }_{A} y, \sigma\right)$ no $i \in N$ prefers the consumption of another agent who receives an object in $A_{i}$. Thus, $\left(x+_{A} y, \sigma\right) \in F_{m+\delta}$. The proof concludes with an argument introduced by Alkan et al. (1991). Since $\delta>0$ can be selected such that $\delta<\varepsilon$, the set $\left\{(y, \sigma) \in F_{m+\delta}: 0 \leq \delta \leq \varepsilon, y \gg x\right\}$ is non-empty. Since preferences are continuous, this set has a maximum. By our claim above, the maximum of this set is necessarily $\varepsilon$.

The Positive Monotonicity Theorem implies the "weak Negative Monotonic-

\footnotetext{
${ }^{7}$ Our restriction to $b \geq 0$ is w.l.o.g.

${ }^{8}$ If $\sigma_{2} \in A \backslash A_{2}$, then $v_{2}\left(l^{n}(\eta), \sigma_{2}\right) \leq v_{2}\left(\delta, \sigma_{2}\right) \leq v_{2}\left(l^{n}(\eta), \mu_{2}\right)$.
} 
ity Theorem," i.e., for each $m \in \mathbb{R}$, each $z \in F_{m}$, and each $\varepsilon>0$, there is an allocation in $z^{\prime} \in F_{m-\delta}$ such that each agent weakly prefers $z$ to $z^{\prime}{ }^{9}$ One can easily see that the Positive and Negative Monotonicity Theorems jointly imply that value-Maxmin, value-Minmax, money-Maxmin, and money-Minmax rules are budget monotone (c.f., Alkan et al., 1991; Velez, 2011; Fujinaka and Wakayama, 2015).

\section{Applications}

\subsection{Reconciling intuitive fairness and budget-monotonicity}

The following are two examples of rules defined by means o intuitive criteria of fairness that violate budget-monotonicity.

Example 1 (A Utilitarian rule may violate budget-monotonicity). Let $N \equiv\{1,2\}$ and $A \equiv\{\alpha, \beta\}$. We construct a preference profile, represented by utility functions $u_{1}$ and $u_{2}$ such that there is no budget-monotone $f$ satisfying that for each $m$,

$$
f(m) \in S_{m} \equiv \underset{z \in F_{m}}{\arg \max } u_{1}\left(z_{1}\right)+u_{2}\left(z_{2}\right) .
$$

Let $0<\varepsilon<\frac{1}{16}, u_{1}\left(x_{\alpha}, \alpha\right) \equiv x_{\alpha}$ for each $x_{\alpha} \in \mathbb{R}, u_{1}\left(x_{\beta}, \beta\right) \equiv x_{\beta}$ for each $x_{\beta} \in \mathbb{R}$, $u_{2}\left(x_{\alpha}, \alpha\right) \equiv x_{\alpha}$ for each $x_{\alpha} \in \mathbb{R}, u_{2}\left(x_{\beta}, \beta\right) \equiv \frac{2 \varepsilon}{1-2 \varepsilon} x_{\beta}+\frac{1}{2(1-2 \varepsilon)}$ for each $x \leq-\varepsilon$, and $u_{2}\left(x_{\beta}, \beta\right) \equiv \frac{1-2 \varepsilon}{2 \varepsilon} x_{\beta}+1$ for each $x_{\beta}>-\varepsilon$ (Fig. 2).

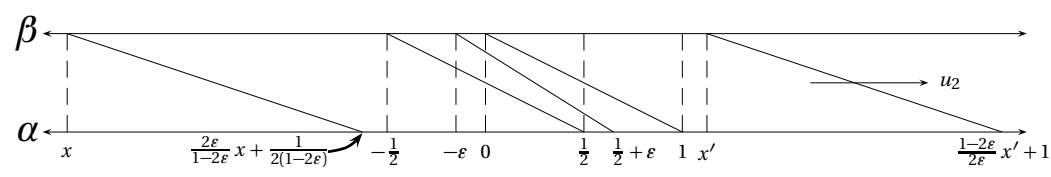

Figure 2: $u_{2}$ in Example 1. Each point $x$ in the axis that.

Let $m \in \mathbb{R}$ and $z \equiv(x, \mu) \in F_{m}$. Since $z$ is efficient, $\mu_{2}=\beta$. Since $z \in F_{m}, a \leq$ $x_{2} \leq m / 2$ where $a$ solves the equation $u_{2}(m-a, \alpha)=u_{2}(a, \beta)$. For each $m$ one can directly calculate $F_{m}$ and characterize its image in utility space, i.e.,

\footnotetext{
${ }^{9}$ Let $t \equiv \sup \left\{s \leq m-\delta: \exists z^{\prime} \in F_{s}, \forall i \in N, z R_{i} z^{\prime}\right\}$. Since preferences are continuous, the Positive Monotonicity Theorem implies that $t=m-\delta$.
} 
$u\left(F_{m}\right)=\left\{\left(u_{1}\left(z_{1}\right), u_{2}\left(z_{2}\right)\right): z \in F_{m}\right\}$ (Fig. 3).

$$
u\left(F_{m}\right)=\left\{\begin{array}{cl}
\operatorname{seg}\left(\left(\frac{m}{2}, \frac{2 \varepsilon}{1-2 \varepsilon} \frac{m}{2}+\frac{1}{2(1-2 \varepsilon)}\right),\left(2 \varepsilon m+\frac{1}{2}, 2 \varepsilon m+\frac{1}{2}\right)\right) & \text { if } m \leq-2 \varepsilon \\
\operatorname{seg}\left(\left(\frac{m}{2}, \frac{1-2 \varepsilon}{2 \varepsilon} \frac{m}{2}+1\right),\left(m+\varepsilon, \frac{1}{2}+\varepsilon\right)\right) \bigcup & \text { if }-2 \varepsilon \leq m \leq \frac{1}{2} \\
\operatorname{seg}\left(\left(m+\varepsilon, \frac{1}{2}+\varepsilon\right),\left(2 \varepsilon m+\frac{1}{2}, 2 \varepsilon m+\frac{1}{2}\right)\right) & \text { if } m \geq \frac{1}{2} \\
\operatorname{seg}\left(\left(\frac{m}{2}, \frac{1-2 \varepsilon}{2 \varepsilon} \frac{m}{2}+1\right),((1-2 \varepsilon) m+2 \varepsilon,(1-2 \varepsilon) m+2 \varepsilon)\right) &
\end{array}\right.
$$

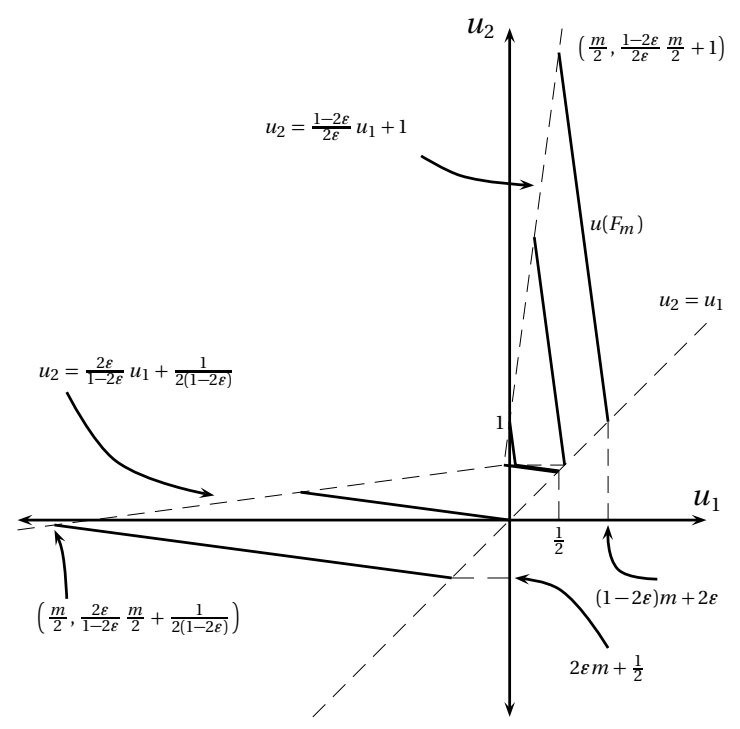

(a)

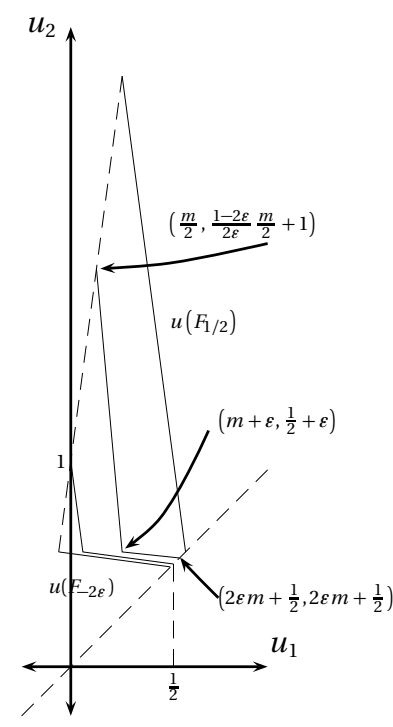

(b)

Figure 3: $u\left(F_{m}\right)$ in Example 1. (a) $u\left(F_{m}\right)$, for $m \leq-2 \varepsilon$ or $m \geq \frac{1}{2}$, (b) Detail of $u\left(F_{m}\right)$, for $-2 \varepsilon \leq$ $m \leq \frac{1}{2}$.

Since $0<\varepsilon<\frac{1}{4}$, the slope of the segments that form $F_{m}$ for $m \leq-2 \varepsilon$ is greater than -1 (segments are flatter than the line with slope -1 ); and the slope of the segments that form $F_{m}$ for $m \geq 1 / 2$ is lower than -1 (segments are steeper than the line with slope -1$)$. Thus, denoting $u\left(S_{m}\right) \equiv\left\{\left(u_{1}\left(z_{1}\right), u_{2}\left(z_{2}\right)\right): z \in S_{m}\right\}$, we can calculate that:

$$
u\left(S_{m}\right)= \begin{cases}\left\{\left(2 \varepsilon m+\frac{1}{2}, 2 \varepsilon m+\frac{1}{2}\right)\right\} & \text { if } m<0 \\ \left\{(0,1),\left(\frac{1}{2}, \frac{1}{2}\right)\right\} & \text { if } m=0 \\ \left\{\left(\frac{m}{2}, \frac{1-2 \varepsilon}{2 \varepsilon} \frac{m}{2}+1\right)\right\} & \text { if } m>0\end{cases}
$$

Let $f$ be such that for each $m \in \mathbb{R}, f(m) \in S_{m}$. Then, for each $\delta \in(0,1 /(1-4 \varepsilon))$, $u_{1}(f(-\delta))>u_{1}(f(\delta))$. Thus, $f$ violates budget-monotonicity. 
Example 2 (An Equal-compensation rule may violate budget-monotonicity). Let $N \equiv\{1,2,3\}$ and $A \equiv\{\alpha, \beta, \gamma\}$. We construct a preference profile $R \equiv\left(R_{i}\right)_{i \in N}$ such that there is no budget-monotone $f$ such that for each $m$,

$$
f(m) \in E_{m} \equiv\left\{z \in F_{m} \text { : for each } z^{\prime} \in F_{m}, \min _{i, j \in N, i \neq j} d_{i j}(z) \geq \min _{i, j \in N, i \neq j} d_{i j}\left(z^{\prime}\right)\right\} .
$$

Let $0<\varepsilon<\frac{1}{5}$ and $R$ a preference profile with numerical representation $u=$ $\left(u_{i}\right)_{i \in N}$, such that: (1) for each $x \in \mathbb{R}, u_{1}(x, \alpha)=u_{1}(x, \beta)=u_{1}(x, \gamma)=x$, (2) for each $i \in\{2,3\}, x \in(-\infty,-1-2 \varepsilon) \cup(-1,0), u_{i}(x, \alpha)=u_{i}(x, \beta)=u_{i}(x+6, \gamma)=x$, (3) $u_{2}(-1-\varepsilon, \beta)>u_{2}(-1-\varepsilon, \alpha)$, (4) $u_{3}(-1-\varepsilon, \alpha)>u_{2}(-1-\varepsilon, \beta),(5) u_{2}(\varepsilon, \beta)=$ $u_{2}(7, \alpha)=u_{2}(7, \gamma)$, and (6) $u_{3}(\varepsilon, \alpha)=u_{3}(7, \beta)=u_{2}(8, \gamma)$ (Fig. 4.)

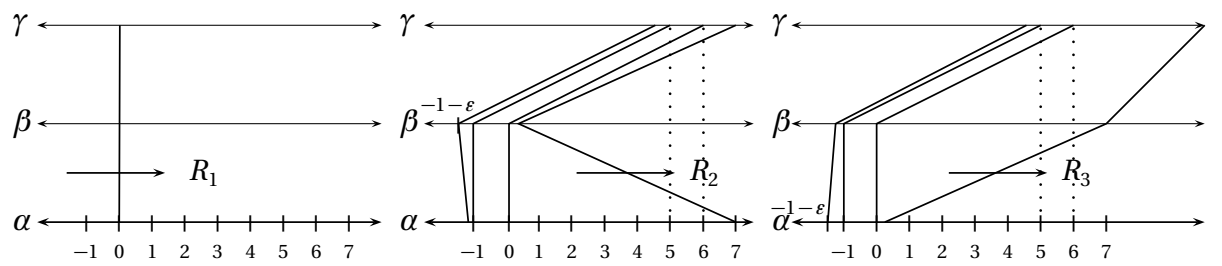

Figure 4: $R$ in Example 2.

Let $(x, \mu) \in F_{0}$. Then, $x_{1} \geq 0$ and $\mu_{1}=\gamma$. We claim that if $(x, \mu) \in E_{0}$, then $x_{1} \geq 2$. To prove this, suppose by contradiction that there is $z \equiv(x, \mu) \in E_{0}$ such that $x_{1}<2$. Then, there is $i \in\{2,3\}$ such that $x_{i}>-1$. Thus, $0 \geq x_{2} \geq-1$ and $0 \geq x_{3} \geq-1$. Since $(x, \mu) \in F, x_{2}=x_{3}>-1$. Thus, $d_{23}(z)=0$. Now, let $z^{\prime}$ be the allocation defined by $z_{1}^{\prime} \equiv(2+2 \varepsilon, \gamma), z_{2}^{\prime} \equiv(-1-\varepsilon, \beta)$, and $z_{3}^{\prime} \equiv(-1-\varepsilon, \alpha)$. One can verify that $z^{\prime} \in F_{0}$ and $\min _{i, j \in N, i \neq j} d_{i j}\left(z^{\prime}\right)>0$. Thus, $z \notin E_{0}$. This is a contradiction.

Let $(x, \mu) \in F_{1}$. Then, $x_{1} \geq 1 / 3$ and $\mu_{1}=\gamma \cdot \sigma(1)=\gamma$. We claim that if $(x, \mu) \in E_{1}$, then $x_{1} \leq 1$. To prove this, suppose by contradiction that there is $z \equiv(x, \mu) \in E_{1}$ such that $x_{1}>1$. Then, there is $i \in\{2,3\}$ such that $x_{i}<0$. Thus, $\min _{i, j \in N, i \neq j} d_{i j}(z) \leq 2 \varepsilon$. Now, let $z^{\prime}$ be the allocation defined by $z_{1}^{\prime}=(1-2 \varepsilon, \gamma)$, $z_{2}^{\prime} \equiv(\varepsilon, \beta)$, and $z_{3}^{\prime} \equiv(\varepsilon, \alpha)$. One can verify that $z^{\prime} \in F_{1}$ and $\min _{i, j \in N, i \neq j} d_{i j}\left(z^{\prime}\right)=$ $1-3 \varepsilon>2 \varepsilon$. Thus, $z \notin E_{1}$. This is a contradiction.

Thus, $f(0) P_{1} f(1)$ and $f$ violates budget-monotonicity.

Examples 1 and 2 illustrate how rules defined on intuitive criteria of fairness may violate budget-monotonicity. It is not necessary to discard all recommendations given by a rule because it violates this property, however. The following 
lemma states that given two recommendations of a non-contestable rule that induce no violation of budget-monotonicity, one can interpolate them with a non-contestable budget-monotone rule.

Lemma 1 (Interpolation of two non-contestable allocations). Let $\{h, l\} \subseteq \mathbb{R}$ be such that $h>l, z \in F_{l}$, and $z^{\prime} \in F_{h}$. Suppose that each agent prefers $z^{\prime}$ to $z$. Then, there is a non-contestable budget-monotone rule $f$ such that $f(l)=z$ and $f(h)=z^{\prime}$.

Proof. Let $h, l, z$, and $z^{\prime}$ as in the statement of the lemma. Let $u \equiv\left(u_{i}\right)_{i \in N}$ be a continuous representation of preferences. Let $v \equiv\left(v_{i}\right)_{i \in N}$ be the representation of preferences given by: for each $\left(x_{\alpha}, \alpha\right)$,

$$
v_{i}\left(x_{\alpha}, \alpha\right)=2+\frac{1}{u_{i}\left(z_{i}^{\prime}\right)-u_{i}\left(z_{i}\right)}\left(u_{i}\left(x_{\alpha}, \alpha\right)-u_{i}\left(z_{i}^{\prime}\right)\right) .
$$

Let $f$ be the value-Maxmin non-contestable rule associated with $v$. Since for each pair $\{i, j\} \subseteq N, v_{i}\left(z_{i}\right)=v_{j}\left(z_{j}\right)=1$ and $v_{i}\left(z_{i}^{\prime}\right)=v_{j}\left(z_{j}^{\prime}\right)=2$, then $f(l)=z$ and $f(h)=z^{\prime}$. By Theorem $1, f$ is budget-monotone.

It is always possible to recover a weaker form of budget-monotonicity in any rule without losing the rule's recommendations for the budgets at which the rule is budget-monotone.

Theorem 3. Let $f$ be a non-contestable rule and $M \subseteq \mathbb{R}$ the set of budgets at which $f$ is budget-monotone. Then, there is a rule $g$ that coincides with $f$ on $M$ and satisfies that for each pair $\{l, h\} \subseteq \mathbb{R}$ such that $h>l$, each agent weakly prefers $g(h)$ to $g(l)$.

Proof. Let $f$ and $M$ be as in the statement of the theorem. Assume without loss of generality that $M \neq \emptyset$, for otherwise the result trivially follows from Theorem 1 . We define rule $g$. For each $m \in M$, let $g(m) \equiv f(m)$. Let $\bar{M}$ be the closure of $M$. Let $m \in \bar{M} \backslash M$ and $\left\{m_{k}\right\}_{k \in \mathbb{N}}$ a sequence in $M$ that converges to $m$. Since $\left\{m_{k}\right\}_{k \in \mathbb{N}}$ is convergent, it is bounded. Thus, $\left\{f\left(m_{k}\right)\right\}_{k \in \mathbb{N}}$ is bounded. Thus, the set of limit points of $\left\{f\left(m_{k}\right)\right\}_{k \in \mathbb{N}}$ is non-empty. Let $g(m) \equiv z$ where $z$ is a limit point of $\left\{f\left(m_{k}\right)\right\}_{k \in \mathbb{N}}$. Let $\{h, l\} \subseteq \bar{M}$ be such that $h>l$. We claim that each agent weakly prefers $g(h)$ to $g(l)$. Let $\left\{h_{k}\right\}_{k \in \mathbb{N}}$ and $\left\{l_{k}\right\}_{k \in \mathbb{N}}$ be two sequences in $M$ such that as $k \rightarrow \infty, h_{k} \rightarrow h, f\left(h_{k}\right) \rightarrow g(h), l_{k} \rightarrow l$, and $f\left(l_{k}\right) \rightarrow g(l)$. Since $h>l$, there is $k \in \mathbb{N}$ such that for each $k \geq K, h_{k}>l_{k}$. Since $f$ is budgetmonotone in $M$, for each $k \geq K, f\left(h_{k}\right)$ is weakly preferred by each agent to $f\left(l_{k}\right)$. Since preferences are continuous, this property is preserved in the limit and each agent weakly prefers $g(h)$ to $g(l)$. If $\bar{M}$ is bounded below, let $g$ coincide on the set of lower bounds of $\bar{M}$ with a non-contestable budget-monotone 
rule that coincides with $g$ at $\min \bar{M}$. If $\bar{M}$ is bounded above, let $g$ coincide on the set of upper bounds of $\bar{M}$ with a non-contestable budget-monotone rule that coincides with $g$ at $\max \bar{M}$. Let $X \equiv\{m \in \mathbb{R} \backslash \bar{M}: \inf \bar{M}<m<\sup \bar{M}\}$. Since $\bar{M}$ is closed, $X$ is an open subset of $\mathbb{R}$. Suppose without loss of generality that $\bar{M}$ is unbounded above and below. Thus, $X$ can be written as the union of pairwise disjoint intervals $\cup_{k \in K}\left(l_{k}, h_{k}\right)$ where $K \subseteq \mathbb{N}$. Let $k \in K$. We define $g$ on $\left(l_{k}, h_{k}\right)$. We claim that $\left\{l_{k}, h_{k}\right\} \subseteq \bar{M}$. Suppose by contradiction, and without loss of generality, that $l_{k} \in \mathbb{R} \backslash \bar{M}$. Thus, there is $k^{\prime} \in K$ such that $l_{k} \in\left(l_{k^{\prime}}, h_{k^{\prime}}\right)$. Thus, $\left(l_{k}, h_{k}\right) \cap\left(l_{k^{\prime}}, h_{k^{\prime}}\right) \neq \emptyset$. This is a contradiction. Let $N_{0} \subseteq N$ be the set of agents who are indifferent between $g\left(l_{k}\right)$ and $g\left(h_{k}\right)$. For each $i \in N_{0}$, and each $m \in\left(l_{k}, h_{k}\right)$, let $g_{i}(m) \equiv g_{i}\left(l_{k}\right)$. Let $\alpha\left(l_{k}\right)$ be the set of objects received by agents in $N_{0}$ at $g\left(l_{k}\right)$ and $\alpha\left(h_{k}\right)$ the set of objects received by agents in $N_{0}$ at $g\left(h_{k}\right)$. By the Decomposition Lemma, $\alpha\left(h_{k}\right)=\alpha\left(l_{k}\right)$. For each $i \in N \backslash N_{0}$, let $g$ coincide on $\left(l_{k}, h_{k}\right)$ with a non-contestable budget-monotone rule, for population $N \backslash N_{0}$ endowed with objects $A \backslash \alpha_{k}$ that interpolates $\left.g\left(l_{k}\right)\right|_{N \backslash N_{0}}$ and $\left.g\left(h_{k}\right)\right|_{N \backslash N_{0}}$. Let $m \in\left(l_{k}, m_{k}\right)$ and $(x, \mu) \equiv g(m)$. By the Decomposition Lemma for each $\alpha \in A \backslash \alpha_{k}, x_{\alpha}$ is less than the consumption of money of the agent who receives $\alpha$ at $g\left(h_{k}\right)$. Since $g\left(h_{k}\right) \in F, g(m) \in F$. It is straightforward to prove that for each pair $\{l, h\} \subseteq \mathbb{R}$ such that $h>l$, each agent weakly prefers $g(h)$ to $g(l)$.

Let $f$ be a non-contestable rule and $M \subseteq \mathbb{R}$ the set of budgets at which $f$ is budget-monotone. It may be impossible to find a non-contestable budgetmonotone rule that coincides with $f$ on $M .{ }^{10}$ A satisfactory approximation is available, however.

Theorem 4. Let $f$ be a non-contestable rule and $M \subseteq \mathbb{R}$ the set of budgets at which $f$ is budget-monotone. Then, for each $\delta>0$ there is $\Delta \subseteq \mathbb{R}$, which can be covered with a set with Lebesgue measure no greater than $\delta$, and a noncontestable budget-monotone rule that coincides with $f$ on $M \backslash \Delta$.

Proof. Suppose without the loss of generality that $M \neq \emptyset$, for otherwise the result trivially follows from Theorem 1 . Let $\bar{M}$ be the closure of $M$ and $X \equiv \mathbb{R} \backslash \bar{M}$. Since $\bar{M}$ is closed, then $X$ is open. Thus, $X$ can be written as the union of pairwise disjoint intervals $\cup_{k \in K}\left(l_{k}, h_{k}\right)$ where $K \subseteq \mathbb{N}$ (here there may be an interval whose lower limit is $-\infty$ and an interval whose upper limit is $+\infty$ ). We claim that for each $k \in K, l_{k} \subseteq \bar{M} \cup\{-\infty\}$ and $h_{k} \subseteq \bar{M} \cup\{+\infty\}$. Suppose by contradiction and without loss of generality that $l_{k} \in \mathbb{R} \backslash \bar{M}$. Then,

\footnotetext{
${ }^{10}$ An example that shows this is a rule $f$ that assigns the same allotment to an agent for an interval say $[l, h]$ but otherwise the welfare of all agents increases with the aggregate budget. Here $M=(-\infty, l) \cup(h,+\infty)$. If $g$ is non-contestable and budget-monotone and coincides with $f$ on $M$, all agents prefers $g(h)$ to $g((l+h) / 2)$. But, $g(h)$ is no better than $f(h)$. Thus, there is no such a $g$.
} 
there are two intervals in $\cup_{k \in K}\left(l_{k}, h_{k}\right)$ that are not disjoint. This is a contradiction. Let $\bar{M}^{*}$ be the set of isolated points in $\bar{M}$, i.e., $m \in \bar{M}$ such that there are $\left\{k, k^{\prime}\right\} \subseteq K$, such that $h_{k}=m$ and $l_{k^{\prime}}=m$. Since $K$ is at most countable, so is $\bar{M}^{*}$. The set $X \cup \bar{M}^{*}$ can be written as a the union of pairwise disjoint intervals $\cup_{k \in K}\left(l_{k}, h_{k}\right)$ where $K \subseteq \mathbb{N}$ and for each $k \in K, l_{k} \in \bar{M} \backslash \bar{M}^{*} \cup\{-\infty\}$ and $h_{k} \in \bar{M} \backslash \bar{M}^{*} \cup\{+\infty\}$. Let $k \in K$. Suppose first that $l_{k}=-\infty$. Then, $h_{k} \in \mathbb{R}$, for otherwise $M=\emptyset$. Then, let $g$ coincide on $\left[l_{k}, h_{k}\right)$ with a non-contestable budget-monotone rule that coincides with $f$ at $h_{k}$. Suppose now that $h_{k}=+\infty$. Then, $l_{k} \in \mathbb{R}$, for otherwise $M=\emptyset$. Then, let $g$ coincide on $\left[l_{k}, h_{k}\right)$ with a noncontestable budget-monotone rule that coincides with $f$ at $l_{k}$. Finally, suppose that $\left\{l_{k}, h_{k}\right\} \subseteq M \backslash \bar{M}^{*}$. Denote the Lebesgue measure by $\lambda$. Let $\varepsilon_{k}$ be the minimum of $\delta / 4^{k}, \lambda\left(\left\{l \in M \backslash \bar{M}^{*}: l \leq l_{k}\right.\right.$, there is no $\left.\left.m \in X \cup \bar{M}^{*}, m \geq l\right\}\right) / 3$, and $\lambda\left(\left\{h \in M \backslash \bar{M}^{*}: h \geq h_{k}\right.\right.$, there is no $\left.\left.m \in X \cup \bar{M}^{*}, m \leq h\right\}\right) / 3$. Since $\left\{l_{k}, h_{k}\right\} \subseteq$ $M \backslash \bar{M}^{*}, \varepsilon>0$. Moreover $\left(l_{k}+\varepsilon, l_{k}\right) \cap M \neq \emptyset$ and $\left(h_{k}, h_{k}+\varepsilon\right) \cap M \neq \emptyset$. Let $l_{k}^{\prime} \in\left(l_{k}+\varepsilon, l_{k}\right) \cap M$ and $h_{k}^{\prime} \in\left(h_{k}, h_{k}+\varepsilon\right) \cap M$. Let $g$ coincide on $\left[l_{k}^{\prime}, h_{k}^{\prime}\right]$ with a non-contestable budget-monotone rule that interpolates $f\left(l_{k}^{\prime}\right)$ and $f\left(h_{k}^{\prime}\right)$. Let $C \equiv \mathbb{R} \backslash \cup_{i \in K}\left[l_{k}^{\prime}, h_{k}^{\prime}\right]$. Since $\cup_{i \in K}\left[l_{k}^{\prime}, h_{k}^{\prime}\right] \supseteq X, C \subseteq \bar{M}$. Since $\bar{M}^{*}$ is countable, then $\lambda\left(\bar{M}^{*}\right)=0$. Thus, $\lambda(\bar{M} \backslash C) \leq \sum_{k \in K} \delta / 2^{k} \leq \delta$. For each $m \in C \cap M$, let $g(m)=f(m)$. For each $m \in C \backslash M$, let $g(m)$ be the limit of a convergent subsequence of $\left\{f\left(m_{k}\right)\right\}_{k \in \mathbb{N}}$ where $\left\{m_{k}\right\}_{k \in \mathbb{N}}$ is a sequence in $M$ that converges to $m$. Since $M \backslash C \subseteq \bar{M} \backslash C$ and $C \cap M=M \backslash(M \backslash C)$, then $g$ coincides with $f$ on the desired set. Since preferences are continuous $g$ is non-contestable. Finally, we prove that $g$ is budget-monotone. Let $m \in C$. We claim that $g$ is budgetmonotone at $m$. Let $h>m$. We claim that each agent prefers $g(h)$ to $g(m)$. Let $l^{\prime} \equiv \min \left\{l_{k}^{\prime}: m \leq l_{k}^{\prime}, k \in K\right\}$. Since $h>m$, there are $\left\{m^{\prime}, m^{\prime \prime}\right\} \in\left(m, l_{k}\right) \cap M$ such that $m^{\prime}<m^{\prime \prime}$. Thus, each agent prefers $g\left(m^{\prime}\right)$ to $g(m)$ and whether $g(h)$ is defined by means of a limit or not, each agent prefers $g(h)$ to $g\left(m^{\prime \prime}\right)$. Since $\left\{m^{\prime}, m^{\prime \prime}\right\} \subseteq C \cap M$, then $f\left(m^{\prime}\right)=g\left(m^{\prime}\right)$ and $f\left(m^{\prime \prime}\right)=g\left(m^{\prime \prime}\right)$. Since $f$ is budgetmonotone on $M$, then each agent prefers $g(h)$ to $g(m)$. A similar argument shows that for each $l<m$, each agent prefers $g(m)$ to $g(l)$. Since the intervals $\left[l_{k}^{\prime}, h_{k}^{\prime}\right]$ are pairwise disjoint, for each $m \in \cup_{i \in K}\left[l_{k}^{\prime}, h_{k}^{\prime}\right], g$ is budget-monotone at $m$.

\subsection{Non-conditional existence of non-contestable allocations with a lower bound or an upper bound on consumptions of money: a characterization}

Our results concerning existence of budget-monotone rules can be used to further our understanding of the conditions necessary for the existence of noncontestable allocations with a minimum or maximum individual consumption 
of money constraint. Think for instance of objects in our model as tasks and consumption of money as salaries with a minimum salary constraint. Think also of objects in our model as rooms and consumptions of money as contribution to rent, with the natural constraint that no roommate is paid to occupy a room.

Two basic results have been previously obtained concerning this problem. First, for a given preference profile, if budget is large enough, there are noncontestable allocations satisfying a given lower bound in consumptions of money (Svensson, 1983, 1987; Maskin, 1987; Alkan et al., 1991; Velez, 2015). Second, whenever all agents are indifferent between receiving any object with no money, for each negative budget (say the rent in the roomates case) there are noncontestable allocations in which each agent's consumption of money is no greater than zero (Su, 1999). These two sets of results are not tight, however. That is, one can find profiles of preferences violating the conditions stated in all these results and for which (i) for each positive budget there are non-contestable allocations in which each agent receives a positive consumption of money, and (ii) for each negative budget there are non-contestable allocations in which each agent receives a negative consumption of money. We state necessary and sufficient conditions guaranteeing that (i) and (ii) above are satisfied. Interestingly, these two conditions are equivalent and hold if and only if there is a noncontestable allocation in which each agent receives an object and no money. This last condition is well known to be characterized as follows: for each set of $k \leq n$ agents, the union of their best objects (i.e., the objects in their best bundles with no money) has at least $k$ objects (Gale, 1960). Our characterization easily follows from a corollary to Theorem 1 that we state first and omit its straightforward proof.

Corollary 1 (Interpolation of one non-contestable allocation). Let $m \in \mathbb{R}$ and $z \in F$. Then there is a non-contestable budget-monotonerule $f$ such that $f(m)=$ $z$.

Proposition 1. The following are equivalent:

1. For each $m \in \mathbb{R}$ such that $m<0$, there is $(x, \mu) \in F_{m}$ such that $x \ll 0$;

2. For each $m \in \mathbb{R}$ such that $m>0$, there is $(x, \mu) \in F_{m}$ such that $x \gg 0$;

3. There is $(0, \mu) \in F_{0}$;

4. For each $S \subseteq N, \mid \bigcup_{i \in S}\left\{\alpha \in A\right.$ : for each $\left.\beta \in A,(0, \alpha) R_{i}(0, \beta)\right\}|\geq| S \mid$.

Proof. From Gale (1960, Theorem 5.2, Pag. 144) there exist a bijection $\mu: N \rightarrow$ $A$ such that for each $i \in N, \mu_{i} \in\left\{\alpha \in A\right.$ : for each $\left.\beta \in A,(0, \alpha) R_{i}(0, \beta)\right\}$, if and 
only if for each $S \subseteq N$, $\left|\bigcup_{i \in S} B_{i}\right| \geq|S|$. Thus, statement 4 is equivalent to statement 3. Suppose that there is $z \equiv(0, \mu) \in F_{0}$. By Corollary 1 , there is $f$ noncontestable and budget-monotone such that $f(0)=(0, \mu)$. Thus, for each $m>0$, $f(m) \in F_{m}$ and each agent prefers $f(m)$ to $f(0)=(0, \mu)$. By the Decomposition Lemma each agent receives a positive consumption of money at $f(m)$. Thus, statement 3 implies statement 2 . By continuity of preferences statement $2 \mathrm{im}$ plies statement 3 . A similar argument shows that statements 1 and 3 are equivalent.

\subsection{Continuity properties of the non-contestable correspondence}

A byproduct of our proof of Theorem 1 is that for each non-contestable and budget-monotone rule the welfare of each agent and for each object the consumption of money associated with the object are all continuous functions of the budget.

Proposition 2. Let $f$ be non-contestable and budget-monotone.

1. Let $w \equiv\left(w_{i}\right)_{i \in N}$ be a continuous representation of $R$. Then, for each $i \in N, w_{i} \circ f_{i}$ is continuous.

2. Let $\alpha \in A$. For each $m \in \mathbb{R}$, let $y_{\alpha}(m) \in \mathbb{R}$ be the consumption of money of the agent who receives object $\alpha$ at $f(m)$. Then, $y_{\alpha}$ is continuous.

Proof. For statement 1 see the proof that statement 1 implies statements 2 and 4 in Theorem 1. For statement 2 see the proof of that statement 1 implies statements 3 and 5 in Theorem 1.

Let $u \equiv\left(u_{i}\right)_{i \in N}$ be a continuous representation of $R$. Our results allow us to better understand the continuity properties of the correspondence that assigns to each budget its possible utility outcomes, i.e., $m \in \mathbb{R} \mapsto u\left(F_{m}\right) \equiv$ $\left\{\left(u_{i}\left(z_{i}\right)\right)_{i \in N} \in \mathbb{R}^{N}: z \in F_{m}\right\} .{ }^{11}$ First, statement 1 in Proposition 2 directly implies that there is always a continuous selection from the correspondence $u(F)$. This is an unusual result for a correspondence that is not convex-valued (Michael, 1956). Second, Corollary 1 and Proposition 2 directly imply that $u(F)$ is lowerhemicontinuous, i.e., for each $m \in \mathbb{R}$, each sequence $\left\{m_{k}\right\}_{k \in \mathbb{N}}$ such that as $k \rightarrow \infty, m_{k} \rightarrow m$, and each $v \in u\left(F_{m}\right)$, there is a sequence $\left\{v_{k}\right\}_{k \in \mathbb{N}}$ such that for each $k \in \mathbb{N}, v_{k} \in u\left(F_{m_{k}}\right)$ and as $k \rightarrow \infty, v_{k} \rightarrow v$.

\footnotetext{
${ }^{11}$ The correspondence $m \in \mathbb{R} \mapsto F_{m}$ may be discontinuous, for an assignment of objects may be sustained in a non-contestable allocation for only a subset of all possible budgets.
} 
Finally, statement 2 in Proposition 2 is useful in the study of the generalization of our model in which there are more objects than agents. Here noncontestable allocations may be inefficient (Alkan et al., 1991). However, one can prove that there are non-contestable and efficient allocations for each possible budget as follows. Suppose that there are $o$ objects and $o>n$. First, introduce $o-n$ agents who value only money. We refer to the economy with $o$ agents as the extended economy. Since the preferences of the fictitious agents are continuous, for each budget, say $m \in \mathbb{R}$, there is an non-contestable allocation in the extended economy with this budget (Alkan et al., 1991; Velez, 2015). Let $z$ be such an allocation. The restriction of this allocation to the "real agents", i.e., $\left(z_{i}\right)_{i \in N}$ is efficient for the initial economy (Alkan et al., 1991). This, does not prove the existence of a non-contestable and efficient allocation in the original economy for budget $m$, because the fictitious agents may receive some consumption of money at $z$. Here is where statement 2 in Proposition 2 comes into play. It directly implies that one can select for each budget a non-contestable allocation in the extended economy in a way that the aggregate consumption of money of the agents in $N$ is a continuous function of the budget. Thus, by a simple fixed point argument, one can show that for each budget, there is a non-contestable and efficient allocation in the economy with $o$ objects and $n$ agents. Moreover, these allocations can be selected so the welfare of each agent is an increasing function of the budget.

\section{Appendix}

Lemma 2 (Decomposition Lemma; Alkan et al. (1991)). Let $z \equiv(x, \mu) \in F$ and $\widehat{z} \equiv(\widehat{x}, \widehat{\mu}) \in F$. Then, both $\mu$ and $\widehat{\mu}$ are bijections between:

(i) $\left\{i \in N: z_{i} P_{i} \widehat{z}_{i}\right\}$ and $\left\{\alpha \in A: x_{\alpha}>\widehat{x}_{\alpha}\right\}$.

(ii) $\left\{i \in N: z_{i} I_{i} \widehat{z}_{i}\right\}$ and $\left\{\alpha \in A: x_{\alpha}=\widehat{x}_{\alpha}\right\}$.

(iii) $\left\{i \in N: \widehat{z}_{i} P_{i} z_{i}\right\}$ and $\left\{\alpha \in A: \widehat{x}_{\alpha}>x_{\alpha}\right\}$.

\section{References}

Alkan, A., 1994. Monotonicity and envyfree assignments. Econ. Theory 4 (4), 605-616.

URL http: //dx.doi .org/10.1007/BF01213627

Alkan, A., Demange, G., Gale, D., 1991. Fair allocation of indivisible goods and criteria of justice. Econometrica 59 (4), 1023-1039.

URL http://www . jstor . org/stable/2938172 
Foley, D., 1967. Resource allocation and the public sector. Yale Econ. Essays 7, $45-98$.

Fujinaka, Y., Wakayama, T., 2015. Maximal manipulation of envy-free solutions in economies with indivisible goods and money. J. Econ. Theory 158, 165185.

URL http://dx.doi.org/10.1016/j.jet.2015.03.014

Gale, D., 1960. The theory of linear economic models. McGraw-Hill.

Kolm, S.-C., 1971. Justice et equité. Paris: Cepremap, (an English translation is: Justice and Equity, 1997, Cambridge, MA: MIT Press).

Maskin, E. S., 1987. On the fair allocation of indivisible goods. In: Feiwel, G. (Ed.), Arrow and the Foundations of the Theory of Economic Policy. NY Univ. Press, pp. 341-349.

Michael, E., 1956. Continuous selections. I. Annals of Math. 63 (2), 361-382. URL http://www.jstor.org/stable/1969615

Moulin, H., Thomson, W., 1988. Can everyone benefit from growth?: Two difficulties. J. Math. Econ. 17 (4), 339-345.

URL http: //dx .doi .org/10 .1016/0304-4068(88)90016-X

Rawls, J., 1972. A theory of Justice. Harvard Univ. Press.

Su, F. E., 1999. Rental harmony: Sperner's lemma in fair division. Amer. Math. Mon. 106 (10), 930-942.

URL http: //www. jstor .org/stable/2589747

Svensson, L.-G., 1983. Large indivisibles: an analysis with respect to price equilibrium and fairness. Econometrica 51 (4), 939-954.

URL http: //www. jstor.org/stable/1912044

Svensson, L.-G., 1987. Erratum to large indivisibles: an analysis with respect to price equilibrium and fairness. Econometrica 55 (2), 489.

URL http: //www.jstor.org/stable/1913253

Tadenuma, K., Thomson, W., 1991. No-envy and consistency in economies with indivisible goods. Econometrica 59 (6), 1755-1767.

URL http: //www. jstor.org/stable/2938288

Tadenuma, K., Thomson, W., 1995. Refinements of the no-envy solution in economies with indivisible goods. Theory and Decision 39 (2), 189-206.

URL http: //dx.doi .org/10 . 1007/BF01078984 
Thomson, W., 1983. The fair division of a fixed supply among a growing population. Math. Oper. Res. 8 (3), 319-326.

URL http: //www. jstor .org/stable/3689303

Thomson, W., 2010. Fair allocation rules. In: Arrow, K., Sen, A., Suzumura, K. (Eds.), Handbook of Social Choice and Welfare. Vol. 2. North-Holland, Amsterdam, New York, Ch. 21.

Thomson, W., 11 2012. On the axiomatics of resource allocation: Interpreting the consistency principle. Econ. Phil. 28, 385-421.

URL http://dx.doi .org/10.1017/S0266267112000296

Varian, H., 1974. Equity, envy, and efficiency. J. Econ. Theory 9, 63-91.

URL http://dx.doi .org/10.1016/0022-0531 (74)90075-1

Velez, R. A., 2011. Are incentives against economic justice? J. Econ. Theory 146 (1), 326-345.

URL http://dx.doi.org/10.1016/j.jet.2010.10.005

Velez, R. A., 2015. Fairness and externalities, Forthcoming Theoretical Econ.

URLhttp://econtheory.org/ojs/index.php/te/article/viewForthcomingFile/1651/125" 